\title{
L'Infinito e il piacere del «contrasto»
}

\section{Selene Sarteschi}

Università degli Studi di Pavia

selene.sarteschi@libero.it

\begin{abstract}
Nell'idillio L'infinito Giacomo Leopardi crea una lirica che, formalmente e contenutisticamente, costituisce il viaggio testuale parallelo a quello fantastico-immaginativo compiuto dal protagonista alla ricerca del piacere. Il poeta appaga la propria tensione al piacere non solo raccontando un'esperienza razionale e sensoriale nei suoi vari passaggi, ma mettendo in pratica la sua tecnica poetica, per comunicare al lettore il diletto della «vera poesia» attraverso il correlato piacere del contrasto, pressoché continuo, fra finito e indefinito al livello fonico, lessicale, sintattico, semantico.
\end{abstract}

Parole chiave: infinito, contrasto, confine, immaginazione, spazio, tempo.

\begin{abstract}
In the idyll «L'infinito» Giacomo Leopardi creates a poem which in its form and content constitutes a textual journey which runs parallel to the fantastic and imaginative one made by the author while looking for pleasure. The poet appeases his own striving for pleasure not only by presenting a rational and sensual experience in its various steps, but also applying his poetical technique in order to convey to the reader the delight of "pure poetry" through the parallel pleasure caused by an almost constant contrast between «finiteness» and «infinity», a contrast shown at the phonic, lexical, syntactic and semantic levels.
\end{abstract}

Key words: infinity, contrast, border, imagination, space, time.

Sempre caro mi fu quest'ermo colle, e questa siepe, che da tanta parte dell'ultimo orizzonte il guardo esclude. Ma sedendo e mirando, interminati spazi di là da quella, e sovrumani silenzi, e profondissima quiete io nel pensier mi fingo; ove per poco il cor non si spaura. E come il vento odo stormir tra queste piante, io quello infinito silenzio a questa voce 
vo comparando: e mi sovvien l'eterno, e le morte stagioni, e la presente e viva, e il suon di lei. Così tra questa immensità s'annega il pensier mio: e il naufragar m’è dolce in questo mare

Celebre, forse la più celebre all'estero, certo la più tradotta, delle poesie leopardiane, L'Infinito ha messo alla prova l'acribia di critici e pensatori, attratti dalla densità di significati e dalla risonanza emotiva e intellettuale racchiuse in un numero così ristretto di versi. Anche attraverso il metro, attraverso pause "forti» ed essenziali, che imprimono al discorso un andamento "a onda", il lettore è sollecitato a vivere o rivivere insieme con il poeta un'esperienza eccezionale, in cui un pensiero mobile riesce a cogliere in un istante la coesistenza di finito e di infinito, di visibile ed invisibile, il dilatarsi e il concentrarsi degli spazi, l'accumulo del tempo. Tutto giocato su una serie di contrasti (stasimovimento, spazio-tempo, passato-presente, finito-infinito, ragione-sentimento, realtà-immaginazione, apertura-chiusura, morte-vita), L'Infinito delinea un processo conoscitivo di singolare efficacia, congiunto a una vigorosa sensazione di piacere e dolcezza, che sono anche piacere e dolcezza di una «nuova» poesia che si viene creando.

Composto probabilmente per primo fra gli idilli realizzati tra il 1819 e il $1821,{ }^{1}$ la cui definizione, nell'ambito della personale esperienza poetica, si deve allo stesso Leopardi, ${ }^{2}$ L'Infinito è, senza dubbio, fra le liriche elaborate in questo torno di tempo, quella in cui più viva si coglie, complice la brevità del testo, l'intenzione dell'autore di tradurre il senso inusitato di un'esperienza dell'animo attraverso un particolarissimo uso dei propri mezzi espressivi: sia sul piano del rapporto metro-sintassi che su quello retorico e linguistico. Se, all'interno della lirica, il rapporto fra metro e sintassi è funzionale a scandire, con le pause interne al verso, più o meno forti (punti, punto e virgola, due punti), le fasi salienti dell'avventura dell'io e a mettere in rilievo, con gli enjambements, il passaggio dalla valenza denotativa a quella infinitivo-evocativa del materiale lessicale, è quindi alla parola in sé che il poeta tende a riconoscere uno specifico statuto emotivo e conoscitivo; perché è la parola che può e vuole proporsi, all'interno di un percorso di creazione-immaginazione («io nel pensier $m i$ fingo $\left.o^{3}[. .].\right)$ ), come l'esplicitazione delle ragioni profonde e dell'essenza stessa del proprio canto. Che la ragione di questo canto sia legata alla

1. Prima di essere accolto nei Canti (1831) L'Infinito apparve per la prima volta nel Nuovo Ricoglitore di Milano nel 1825; quindi nell'anno successivo fu pubblicato nell'opuscolo dei Versi stampato a Bologna.

2. Leopardi ci offre la seguente definizione: «Idilli esprimenti situazioni, affezioni, avventure storiche del mio animo", in Giacomo LEOPARDI, Tutte le opere (TO), con introduzione e cura di Walter BINNI, con la collaborazione di Enrico GHIDETTI, 2 voll., Firenze: Sansoni, 1969; la citazione proviene dal vol. I, p. 372.

3. Cfr. «Falsissima idea considerare e definir la poesia per arte imitativa, metterla colla pittura ec. Il poeta immagina: l'immaginazione vede il mondo come non è, si fabbrica un mondo che non è, finge, inventa, non imita, non imita (dico) di proposito suo: creatore, inventore, 
testimonianza di un'autoremunerazione attraverso il piacere lo spiega Leopardi stesso in un appunto dello Zibaldone del 1 agosto 1821:

Circa le sensazioni che piacciono pel solo indefinito puoi vedere il mio idillio sull'Infinito, e richiamar l'idea di una campagna arditamente declive in guisa che la vista in certa lontananza non arrivi alla valle; e quella di un filare d'alberi, il cui fine si perda di vista, o per la lunghezza del filare, o perch'esso pure sia posto in declivio ec. ec. ec. Una fabbrica una torre ec. veduta in modo che ella paia innalzarsi sola sopra l'orizzonte, e questo non si veda, produce un contrasto efficacissimo e sublimissimo tra il finito e l'indefinito ec. ec. (1430-31; miei i corsivi, eccetto il secondo).

Da questo brano emerge che il piacere è legato ad una sensazione particolare: quella che nasce dal "contrasto» fra due diversi stati o condizioni percettive. Nel caso specifico del passo dello Zibaldone, il contrasto si attiva perché, a un certo punto, la vista non può proseguire; mentre è in atto la percezione visiva e si prospettano una campagna, un filare d'alberi, una fabbrica, una torre, d'un tratto si verifica un'interruzione nella possibilità di andare oltre quella particolare veduta paesaggistica. L'impedimento, naturale o artificiale, che ostacola il continuum della percezione visiva più in là di un certo limite determina dunque una sorta di momentanea stasi nella rappresentazione mentale del paesaggio circostante: ed è proprio nell'attimo del «blocco» della facoltà percettiva, legato all'occasionale perdita del concetto di confine (ad esempio la temporanea scomparsa dell'orizzonte coperto dalla torre), che può subentrare un altro tipo di esperienza sensitiva, con l'istantaneo, fantastico-immaginifico superamento dell'ostacolo che induce la particolare sensazione del "contrasto efficacissimo e sublimissimo fra il finito e l'indefinito". L'infinito altro non può essere che il prolungarsi di questo contrasto, di questo sbilanciamento verso un punto di fuga mentale: un errare di là da ogni confine intellettivo, ove non si riconosce più l'esistenza di un limite valido a circoscrivere le percezioni del proprio animo.

Un anno prima di stendere il passo dello Zibaldone sopra riportato Leopardi aveva già affidato alla medesima opera una serie di riflessioni intorno "alla inclinazione dell'uomo all'infinito». Si tratta di un lungo brano — steso nel luglio 1820 - in cui il poeta si sofferma sull'innata tendenza al piacere:

Veniamo alla inclinazione dell'uomo all'infinito. Indipendentemente dal desiderio del piacere, esiste nell'uomo una facoltà immaginativa, la quale può concepire le cose che non sono, e in un modo in cui le cose reali non sono. Considerando la tendenza innata dell'uomo al piacere, è naturale che la facoltà immaginativa faccia una delle sue principali occupazioni della immaginazione al piacere. E stante la detta proprietà di questa forza immaginativa, ella può

non imitatore; ecco il carattere essenziale del poeta». (Zibaldone, 23...29 Ago. 1828, 4358), in Giacomo LeOPARDI, Zibaldone di pensieri, a cura di Giuseppe PACELLA, 3 voll., Milano: Garzanti, 1991. Sempre miei i corsivi se non indicato diversamente. 
figurarsi dei piaceri che non esistano, e figurarseli infiniti 1. in numero, 2. in durata, 3. e in estensione. [...] Del rimanente alle volte l'anima desidererà ed effettivamente desidera una veduta ristretta e confinata in certi modi, come nelle situazioni romantiche. La cagione è la stessa, cioè il desiderio dell'infinito, perché allora in luogo della vista, lavora l'immaginazione e il fantastico sottentra al reale. L'anima s'immagina quello che non vede, che quell'albero, quella siepe, quella torre gli nasconde, e va errando in uno spazio immaginario, e si figura cose che non potrebbe se la sua vista si estendesse da per tutto, perché il reale escluderebbe l'immaginario. Quindi il piacere ch'io provava sempre da fanciullo, e anche ora nel vedere il cielo ec. attraverso una finestra, una porta, una casa passatoia, come la chiamano. Al contrario la vastità e molteplicità delle sensazioni diletta moltissimo l'anima. Ne deducono ch'ella è nata per il grande ec. Non è questa la ragione. Ma proviene da ciò, che la moltiplicità delle sensazioni confonde l'anima, gl'impedisce di vedere i confini di ciascheduna, toglie l'esaurimento subitaneo del piacere, la fa errare d'un piacere in un altro senza poterne approfondare nessuno, e quindi si rassomiglia in certo modo a un piacere infinito. (Zibaldone, 167-171).

Non possono sussistere dubbi sull'attinenza di questo passo con L'Infinito. Se poi allarghiamo appena il contesto del primo brano trascritto dello Zibaldone, del 1 agosto 1821 , possiamo ricostruire le linee portanti dell'idillio: ${ }^{4}$

Lantico è un principalissimo ingrediente delle sublimi sensazioni, siano materiali, come una prospettiva, una veduta romantica ec. ec. o solamente spirituali ed interiori. Perché ciò? per la tendenza dell'uomo all'infinito. L'antico non è eterno, e quindi non è infinito, ma il concepire che fa l'anima uno spazio di molti secoli, produce una sensazione indefinita, l'idea di un tempo indeterminato, dove l'anima si perde, e sebben sa che vi sono confini non li discerne, e non sa quali sieno. (Zibaldone, 1429).

La molteplicità delle sensazioni e le sensazioni indefinite producono piacere "per la tendenza dell'uomo all'infinito". Ne deriva che il contenuto dell'idillio può essere definito come il racconto di un viaggio immaginario, lungo le cui tappe l'io, nel percorso di andata, prima si smarrisce nella vastità e lontananza dello spazio, poi, in quello di ritorno, attraverso lo stimolo di una percezione uditiva, si rinviene nella consistenza di un flusso temporale che, dopo essersi dilatato, si restringe di nuovo dal remoto passato al presente: originando così l'urto, ossia il «sublimissimo contrasto», fra «finito» e «indefinito». L'antitesi fra queste due dimensioni percepite dall'animo che perdura a vaga-

4. Ma osserva giustamente Santagata: «Si noti però che tutti i pensieri leopardiani sull'infinito sono posteriori alla composizione dell'idillio. Questo è nato da una intuizione, apre la strada alle riflessioni del suo autore, non ne dipende». Marco SANTAGATA, «Lettura didattica dell'"Infinito"”, p. 901-909, in Studi in onore di Pier Vincenzo Mengaldo per i suoi settant'anni, a cura degli allievi padovani, vol. I, Firenze: SISMEL, Edizioni del Galluzzo, 2007 (citaz. da p. 904). 
re in una sensazione di piacere, rappresenta al pensiero l'idea dell'immensità che non è solo quella dello spazio e del tempo, ma anche la percezione del proprio sconfinato sommovimento interiore che fluttua fra diverse forme di piacere: in cui il pensiero stesso si immerge e si sprofonda per gustarne l'essenza. Dalla consapevolezza di essere vivo e "presente», pronto a cogliere il pulsare dell'esistenza nel suo percepibile e inconfondibile "suon[o]», giunge a compimento, sottolineato anche dalla perfetta coincidenza fra metro e sintassi, il viaggio della mente nell'infinito.

In una nota dello Zibaldone dell'agosto 1821, prendendo spunto da un'affermazione di Cicerone, il poeta sostiene, in perfetto accordo con la sua visione filosofica, la diversità dell'uomo moderno rispetto all'antico nel modo di provare l'inclinazione all'infinito:

Dice Cicerone [...] che gli uomini di gusto nell'eloquenza non si appagano mai pienamente né delle loro opere né delle altrui, e che la mente loro semper divinum atque infinitum desiderat, a cui le forze dell'eloquenza non arrivano. Questo detto è notabilissimo riguardo all'arte, alla critica, al gusto. $\mathrm{Ma}$ ora lo considero in quanto ha relazione a quel perpetuo desiderio e scontentezza che lasciano, siccome tutti i piaceri, così quelli che derivano dalla lettura, e da qualunque genere di studio; ed in quanto si può riferire a quella inclinazione e spasimo dell'uomo verso l'infinito, che gli antichi, anche filosofi, poche volte e confusamente esprimono, perché le loro sensazioni essendo tanto più vaste e più forti, le loro idee tanto meno limitate e definite dalla scienza, la loro vita tanto più vitale ed attiva, e quindi tanto maggiori le distrazioni de' desiderii, che la detta inclinazione e desiderio non potevano sentirlo in un modo così chiaro e definito come noi lo sentiamo. (Zibaldone, 27 Agosto 1821, 1573-74; nel testo il primo e l'ultimo corsivo).

E immediatamente dopo il poeta, restando nel merito del piacere che proviene dallo studio e dalla lettura, aggiunge:

Osservo però che non solo gli studi soddisfano più di qualunque altro piacere, e ne dura più il gusto, e l'appetito ec. ma che fra tutte le letture, quella che meno lascia l'animo desideroso del piacere, è la lettura della vera poesia. La quale destando mozioni vivissime, e riempiendo l'animo di idee vaghe e indefinite e vastissime e sublimissime e mal chiare ec. lo riempie quanto più si possa a questo mondo. Così che Cicerone non avrebbe forse potuto dire della poesia ciò che disse dell'eloquenza. (Zibaldone, 1574-75).

È dunque la creazione dell'Infinito come lirica in sé perfettamente compiuta che costituisce il viaggio testuale parallelo a quello fantastico-immaginifico del protagonista alla ricerca del piacere. Proveremo, a questo punto, a verificare in quale modo Leopardi appaghi la propria tensione al piacere non soltanto raccontando un'esperienza razionale e sensoriale, nei suoi vari passaggi, ma mettendo in pratica, sulla pagina scritta, la sua tekne poetica, per comunicare al lettore il diletto "della vera poesia» attraverso il correlato piacere del 
"contrasto", pressoché continuo, fra finito (chiusura) e indefinito (apertura), a vari livelli: ${ }^{5}$ fonico, lessicale, sintattico, contenutistico.

Passeggiando non lontano dalla casa paterna il giovane Leopardi era solito recarsi sul monte Tabor «che signoreggia la valle sottoposta e tutta la Marca occidentale fino agli Appennini...a' tempi del poeta era veramente ermo, folto di alberi e irto di sterpi a maniera di siepi» (Mestica). ${ }^{6}$ Colle «caro» al poeta poiché compagno nella sua solitudine: «O graziosa luna, io mi rammento / che, or volge l'anno, sovra questo colle / io venia pien d'angoscia a rimirarti» (Alla luna, vv. 1-3).

L'incipit dell'Infinito si apre su una notazione affettiva ed intensa, sottolineata dall'avverbio "Sempre» che indica lunga durata temporale, ${ }^{7}$ rafforzata dall' unico verbo al passato presente nell'idillio, «fu»; tale passato, infatti, invece di fissare un momento preciso nella dimensione temporale lo diluisce nella vastità di un continuum emotivo senza limite. Quel «sempre, del resto, sembra presupporre un silenzioso colloquio interiore che affonda le proprie radici in un tempo lontano e che solo a un certo momento si traduce in parola, come se quell'amalgama di sentimento e pensiero all'improvviso precipitasse e sfociasse con sorprendente naturalezza nella scelta di pochi, ma essenziali, oggetti comuni, familiari. ${ }^{8}$ Il primo verso, inoltre, è caratterizzato da una lieve inversione sintattica che pone in primo piano il principale aggettivo ("caro») riferito al soggetto (il «colle») che chiude, invece, preceduto dal dimostrativo

5. Ibid., p. 902: «Se sul piano tematico l'“effetto infinito" scatta in presenza di un ostacolo, su quello delle forme l'"effetto infinitivo" (Blasucci) si potenzia in presenza di segnali di chiusura». Lo studioso rimanda a Luigi BLASUCCI, Leopardi e i segnali dell'infinito, Bologna: Il Mulino, 1985.

6. Prelevo la citazione da Giacomo LeOpard, Canti, a cura di Niccolò Gallo e Cesare GarBOLI, Torino: Einaudi, 1993, p. 105. Corsivo nel testo.

7. Insiste molto su questo aspetto durativo del «Sempre» Enrico FENZI, nell'articolo davvero importante "Note di lettura all'"Infinito"», in $\Lambda A \Theta E$ BI $\Sigma A \Sigma$, Ricordando Ennio S. Burioni, a cura di Renato GENDRE, Alessandria: Edizioni dell'Orso, 1998, p. 99-127 (vd. in partic. p. 99-101).

8. Nel Discorso di un italiano, Leopardi contrappone all'interesse dei romantici per la «singolarità» la predilezione classica per il familiare, l' «oggetto comune» dei poeti antichi. Giacomo LEOPARDI, Discorso di un italiano intorno alla poesia romantica, in TO, op. cit., p. 914-948: «Imperocché allora è grandissima l'efficacia della poesia, quando l'imitazione è rara, l’oggetto comune. [...] Ora l'efficacia di questa poesia che sola è propriamente poesia, la doveano sentire gli antichi meglio di noi, come sappiamo che facevano, imperocché un tempo furono affatto ordinari in essa tutti e due quegl'inestimabili accidenti, la rarità dell'imitazione e la familiarità degli oggetti» (p. 926-27). E ugualmente, nei primi appunti dello Zibaldone risalenti grosso modo allo stesso periodo del Discorso, Leopardi sostava a lungo sul modo di comporre degli antichi, opposto a quello dei moderni: «Or dunque bisogna eccitare questo patetico, questa profondità di sentimento nei cuori: e qui, com'è naturale, consisterà la somma arte del poeta. E qui è dove il Breme e tutti quanti i romantici e i chateaubriandisti ec. ec. scappano di strada. Che cosa è che eccita questi sentimenti negli uomini? La natura, purissima, tal qual è, tal quale la vedevano gli antichi: le circostanze, naturali, non proccurate mica a bella posta, ma venute spontaneamente: quell'albero, quell'uccello, quel canto, quell'edifizio, quella selva, quel monte, tutto da per se, senz'artificio [...]» (Zibaldone, Ago. 1817...Dic. 1818, 15-16). 
"quest[o] e dal secondo aggettivo «ermo", l'endecasillabo, perfettamente autonomo sintatticamente, al pari dell'ultimo. Ed è proprio nell'incipit e nell'explicit, i soli versi di senso compiuto dell'Infinito, che si trovano le due uniche affermazioni affettive presenti nell'idillio, quasi a volerlo chiudere circolarmente fra un tenero tributo ed un piacevole moto dell'animo. La lievissima inversione sintattica del primo verso, atta ad assecondare l'affettuoso sentimento del poeta verso il colle, dà origine all'elegante sinuosità del componimento ${ }^{9} \mathrm{e}$ insieme preannuncia lo scarto tra la sospensione del cuore (v. 8), e il «suon[o]» vitale della stagione presente (v. 13) che contrassegnano, rispettivamente, con la seconda e la quarta pausa forti rinvenibili nellidillio (si tratta in entrambi i casi di un punto fermo che divide a metà l'endecasillabo), l'incremento emotivo in cui culminano due momenti cruciali dell'avventura dell'io.

Focalizzerò per il momento l'attenzione sui tre versi introduttivi, così tradizionali, ${ }^{10}$ per sottolineare come sia proprio con quest'esordio che Leopardi predisponga il lettore a cogliere la valenza «infinitiva» ${ }^{11}$ del suo racconto e il "contrasto efficacissimo e sublimissimo tra il finito e l'indefinito». Ė in questa sezione iniziale dell' Infinito che il poeta inserisce cinque sostantivi che, secondo uno schema di 2 (colle, siepe $)+2$ (parte, orizzonte) +1 (guardo), si ripartiscono lo spazio semantico. Sia la prima che la seconda coppia di parole è dislocata in due endecasillabi diversi: rispettivamente primo/secondo, secondo/terzo, ed è proprio fra il secondo e il terzo di questi endecasillabi che si registra la prima infrazione metrico-sintattica della lirica, l'enjambement che interrompe il senso compiuto del costrutto verbale, separando non tanto il sostantivo "parte» dalla preposizione «dell'», ma soprattutto il sintagma «tanta parte» da quello successivo: «ultimo orizzonte». Il primo sintagma è costituito da due parole bisillabe («tanta parte») con accento principale sulla vocale chiara ed aperta «a», mentre il secondo è formato da una coppia di termini («ulti-

9. Marco SAnTAGATA, «Lettura didattica [...]», art. cit., p. 902: «Ma dopo il punto fermo del v. 3, l'unico a fine di verso di tutta la poesia (con l'ovvia eccezione dell'ultimo), subentra un procedere franto nella sintassi, variato nel ritmo e tuttavia fluido, scorrevole e continuato». Credo tuttavia che la fluidità e scorrevolezza dell' Infinito nasca prima del punto fermo del verso 3, e che inizi proprio con il primo verso.

10. Solo per fare un esempio vedi ibid., p. 901-902: «Il lettore a cui fossero capitati fra le mani freschi di stampa il fascicolo della rivista o il volumetto, imbattutosi nell' Infinito e letti i primi tre versi, avrebbe potuto ritenere di trovarsi di fronte al solito quadretto campestre di gusto arcadico-petrarchesco. Se però avesse proseguito nella lettura ben presto si sarebbe dovuto ricredere. In effetti, superata l'avversativa del v. 4 ("Ma sedendo e mirando..."), la poesia cambia aspetto». Oppure, Luigi BLASUCCI, "Paragrafi sull'“Infinito"», p. 97-122, in Leopardi e i segnali dell'infinito", op. cit.: "È stato finemente osservato da Fubini il carattere di "andante regolare" dei primi tre versi, "leggendo i quali sentiamo riecheggiare qualche inizio petrarchesco [...]". È vero che quell'"andante" è movimentato dall'enjambement dei vv. 2-3; ma questo motivo può considerarsi a sua volta assorbito nel giro del terzo verso, chiuso da un punto fermo: sicché nell'insieme i primi tre versi possono effettivamente suggerire l'idea di un primo piede di una fronte di canzone», p. 99-100.

11. Rinvio immediatamente, sui "procedimenti di attivazione del motivo dell'infinito" nei Canti, a due fondamentali contributi di Luigi BLASUCCI, "Paragrafi sull'"Infinito"», art. cit., e "I segnali dell'infinito", p. 123-151, in Leopardi e i segnali dell'infinito, op. cit. 
mo orizzonte») di cui il primo sdrucciolo con accento sulla vocale più scura $\mathrm{e}$ chiusa "u», l'altro quadrisillabo che completa, con «o», «i», «e», la scala delle vocali. Il suono delle quattro parole, («tanta», "parte», «ultimo», "orizzonte») attiva, con la sequenza di «a», «e», «u», «i», «O», con l'allitterazione della consonante «t», con la nota ribattuta «ta» (n)«ta» e la presenza ravvicinata delle due consonanti liquide «rt»-«lt» («parte»-«ultimo») un processo di dilatazione sul piano acustico, già in atto nel momento in cui l'ultima sezione del verso 3, «il guardo esclude», giunge ad ampliare ulteriormente il significato di quanto precede: tanto sul piano visivo, perché il verbo «escludere» dà ragione del «contrasto» fra "chiusura» vs «apertura» dello sguardo, ancora implicito, quanto su quello nuovamente acustico, poiché "guardo» ed «esclude» ripropongono le due medesime vocali accentate, la «a» e la «u» che si situano agli estremi della tastiera fonica delle vocali. L'opposizione fra «apertura» e «chiusura» potenzia l'effetto infinitivo che è già in funzione nei primi tre endecasillabi dell'idillio.

La carica semantica di ciò che è separato dall'enjambement (tanta parte / ultimo orizzonte) risulta incrementata: tant'è che l'arricchimento del significato investe a ritroso anche il sintagma «questa siepe»: l'oggetto concreto che, assieme al colle, è altrettanto caro al poeta poiché costituisce l'ostacolo che impedisce allo sguardo di percepire quanto si vedrebbe a perdita d'occhio se questa non ci fosse, se non determinasse in potenza quell'ormai noto «contrasto» fra finito e indefinito. La siepe ostacola dunque, nella sua consistenza materiale e nella realtà effettuale, la continuità dello sguardo, anticipando quanto avviene a livello metrico-sintattico con la presenza dell'enjambement che, a sua volta, ostacola il compimento semantico del verso 2 . La siepe che preclude allo sguardo "tanta parte» dell'orizzonte terrestre è il primo motore che predispone l'avventura dell'io che sta per inoltrarsi nell'infinito. Ma prima di procedere oltre soffermiamoci ancora un momento sui due sintagmi «tanta parte» e "ultimo orizzonte» separati dall'enjambement. Rispettivamente nel secondo e nel terzo endecasillabo, "tanta» e "ultimo" sono due termini che compaiono, raggruppati da Leopardi con altri, nell' Indice del mio Zibaldone, sotto la comune definizione di «Voci e frasi piacevoli e poetiche assolutamente, per l'infinito o indefinito del loro significato ec.». Abbiamo appena visto che «tanta» è bisillabo, "ultimo» è trisillabo, mentre il sostantivo "orizzonte», pur non rientrando fra le parole che il poeta qualifica esplicitamente come indefinite nel significato appartiene comunque alla serie dei vocaboli che semanticamente contengono l'idea di infinito: e non solo sul piano del significato, ma anche su quello del significante, ossia del puro aspetto fonico, sonoro. Infatti «orizzonte» è una parola quadrisillaba che per la sua «temporis occupatio» si presenta ampia, vasta, dilatata. Fra le parole indefinite raggruppate nell' Indice zibaldoniano e presenti nell' Infinito si trovano anche «ermo" (v. 1); «silenzio" (al plurale al v. 6, al singolare al v. 10); "profondo" ("profondissima», v. 6); «eterno» (v. 11); «morte» (v. 12), sì da coprire quasi interamente lo spazio testuale dell'idillio. Armonizzate a queste parole si trovano termini polisillabi (pentasillabi o quadrisillabi) come il già visto «orizzonte», gli aggettivi «interminati» (v. 4), «sovrumani» (v. 5); i sostantivi «infinito» (v. 10); «immen- 
sità» (v. 14); il verbo «naufragar» (v. 15). La loro valenza evocativa e infinitiva è messa in risalto dalla presenza della maggior parte delle parole della poesia che, per opposizione, si caratterizzano come prive del requisito dell'infinità. Nel fluire dei versi si crea, dunque, un continuo contrappunto nella direzione del «contrasto» fra finito e indefinito.

Il verso 4 si apre con un "Ma»: ${ }^{12}$ la breve particella, che imprime un movimento centrifugo al discorso — l'io si proietterà nello spazio dal luogo circoscritto del colle verso l'esterno, dalla chiusura verso la massima apertura, dal finito verso l'indefinito - introduce i due gerundi assoluti, «sedendo e mirando", adoperati per amplificare l'evento che sta per essere descritto. Trovandosi lì, dietro la siepe, il poeta contempla quanto lo circonda e, nel suo «mirare», ${ }^{13}$ lo sguardo oltrepassa la linea di confine che la siepe delimita: "di là da quella» (v. 5) il pensiero può vagare e creare nell'immaginazione un'idea che si espande in sequenza. L'io concepisce, o meglio «si finge» nel pensiero «interminati / spazi», «e sovrumani / silenzi, e profondissima quiete» (vv. 4-6). ${ }^{14}$ Si noti l'accentuazione forte sul timbro vocalico aperto «a» nella sequenza «interminàti»«spàzi»-«sovrumàni» che dilata ulteriormente il significato vasto e indefinito caratteristico di questi termini.

I primi due intensi enjambements ai versi 4-5 e 5-6, rafforzano la catena del duplice polisindeto e isolano la potenza semantica di «interminati» e «sovrumani» in punta di verso, determinando un vortice semantico in cui il soggetto precipita sopraffatto dall'immensità degli «spazi» e dei soprannaturali «silenzi» che si collegano: per fondersi, nell'ulteriore estensione del legame polisindetico, in una "quiete» che nella sua superlativa profondità e vastità, enfatizzata dalla dieresi, contrassegna la climax del processo fantasmatico. In questa fantasia nella quale dominano «spazi», «silenzi», "quiete» incommensurabili — dilatate a dismisura dagli aggettivi che li qualificano- il cuore, sostituto metonimico dell'io, giunge quasi, tale è il significato di "per poco», a provare timore. La pausa forte costituita dal punto fermo divide in due parti l'endecasillabo, a segnare un "disaccordo» imprevisto nel rapporto metro-sintassi. Proviamo a ripercorrere più da vicino quanto è accaduto all'io a partire dalla congiunzione «Ma».

Verificata l'impossibilità di proseguire con lo sguardo oltre il delimitato spazio consolatorio e rasserenante che lo circonda, gli occhi del poeta conti-

12. Enrico FENZI, «Note di lettura all'“Infinito”», in $\Lambda A \Theta E$ BI $\Sigma A \Sigma$, Ricordando Ennio S. Burioni, art. cit.: "Questo $M a$ è una sorta di concentrato ossimoro, perché in prima istanza, ha un valore oppositivo quanto consequenziale». Per l'articolato discorso dello studioso intorno al «Ma» e la discussione critica relativa vd. p. 103-104.

13. Il significato di questo "mirare» contiene in sé il polisemico valore di un semplice "guardare» che a poco a poco si trasforma in un "ammirare», in un "estasiarsi» dinanzi alla vista che, da esteriore, diviene interiore.

14. L'aggettivo «interminati» è hapax nei Canti, al pari di "profondissima», mentre "sovrumani» vi ricorre ancora tre volte, una al plurale, al verso 28 di Sopra il ritratto di una bella donna: "di sovrumani fati», due al singolare, al verso 124 del Risorgimento: "voi, raggio sovrumano» e al verso 208 della Palinodia: "Oh menti, ho senno, oh sovrumano acume». 
nuano a fissare la siepe: sino a oltrepassarne il confine. Il «Ma» segna il punctum temporis in cui lo sguardo non solo vede, ma contempla. La congiunzione "Ma», con il suo significato avversativo — «invece», "tuttavia», "al contrario" rispetto a quanto è accaduto sino ad ora - segna il trapasso dal tempo presente, che l'io si lascia alle spalle, verso ciò che è illimitato nella dimensione spaziale dell'immediato futuro. E per l'innata tendenza dell'uomo all'infinito e al piacere l'io comincia a "fingere», ossia a creare nella propria mente, "la maggiore operazione della più fervida e più delirante e sognante immaginativa» (Zibaldone, 29-30 agosto 1823, 3309). La vastità dell'attività fantastica oltre il limite spaziale rappresentato dalla «siepe» e quello temporale rappresentato dal «Ma» è tale e tanta da presentarsi sotto una triplice forma di infinito, lungo la sequenza di tre complementi oggetti che precedono sintatticamente il soggetto che agisce: «io nel pensier mi fingo [...]» (v. 7), col risultato di un'amplificazione delle immagini poetiche «indefinite» e di una mise en relief delle rappresentazioni fantasticate rispetto al loro creatore. Scatta a questo punto, dopo l'amplificazione, la «chiusura», la pausa interna del punto e virgola a metà del verso 7 , preannuncio dell'immediata interruzione ancora più forte rappresentata dal punto fermo, a metà dell'endecasillabo successivo. Sia il verso 7 che il verso 8 si aprono con l'evocazione del soggetto poetante: da una parte l'io che ha originato con la sua finzione gli spazi sconfinati, dall'altra il cuore, sostituto metonimico dell'io, che in quello spazio («ove», v. 7) è venuto a trovarsi, sino a «spaurirsi» (v. 8). ${ }^{15}$ Prendiamo in esame i due brani seguenti:

Anzi è notabile che l'anima in una delle dette estasi, vedendo p.e. una torre moderna, ma che non sappia quando è fabbricata, e un'altra antica della quale sappia l'epoca precisa, tuttavia è molto più commossa da questa che da quella. Perché l'indefinito di quella è troppo piccolo, e lo spazio, benché i confini non si discernano, è tanto angusto, che l'anima arriva a comprenderlo tutto. Ma nell'altro caso, sebbene i confini si vedano, e quanto ad essi non vi sia indefinito, v'è però in questo, che lo spazio è così ampio che l'anima non l'abbraccia, e vi si perde. (Zibaldone, 1 Agosto 1821, 1430).

E nel modo che di grandissimi regni ed imperi umani, e loro maravigliosi moti, che furono famosissimi in altre età, non resta oggi segno né fama alcuna; parimente del mondo intero, e delle infinite vicende e calamità delle cose create, non rimarrà pure un vestigio; ma un silenzio nudo, e una quiete altissima empieranno lo spazio immenso. (Cantico del gallo silvestre). ${ }^{16}$

15. Per una spiegazione puntuale dei vv. 7-8 dell'Infinito, in rapporto al decimo libro del trattato Del sublime pseudo-longiniano che contiene l'ode saffica cui Leopardi si sarebbe ispirato nel comporli, rimando al bellissimo contributo di Gilberto LONARDI, «Leopardi, Saffo, il Sublime», p. 57-92 (in partic. p. 74-77), in L'oro di Omero, L'«Iliade», Saffo: antichissimi di Leopardi, Venezia: Marsilio, 2005.

16. Giacomo LeOPARDI, Operette morali, in TO, op. cit., vol. I, p. 158. Nel Cantico si incontra anche il sintagma "profondissima quiete», ivi, p. 156. 
Nel passo del Cantico del gallo silvestre, senza sfumature di indeterminatezza o di dolcezza, ritroviamo all'interno di un'unica proposizione i tre sostantivi dell' Infinito, anche se in diverso ordine: il «silenzio nudo ${ }^{17}$ corrisponde a «sovrumani / silenzi», la «quiete altissima» alla "profondissima quiete», lo «spazio immenso» a «interminati / spazi». Nell'idillio l'uso del plurale è finalizzato a rendere le parole maggiormente poetiche: «Le parole che indicano moltitudine, copia, grandezza, lunghezza, larghezza, altezza, vastità ec. ec. sia in estensione, $\mathrm{o}$ in forza, intensità ec. ec. sono pure poeticissime, e cos̀ le immagini corrispondenti. [...]» (Zibaldone, 3 Ott. 1821, 1825). Fra tali parole "poeticissime» si trova anche "profondo" (Zibaldone, 28 Sett. 1821, 1798), e questa circostanza spiega la poeticità, resa più intensa dall'uso del superlativo, del sintagma "profondissima quiete».

$\mathrm{Ma}$ è il brano dello Zibaldone che può aiutarci a intendere il significato dell'endecasillabo ricomponibile a cavallo dei versi 7-8: «ove per poco / il cor non si spaura». La ripetizione insistita e ravvicinata della vocale «o» crea una figura fonica che oltrepassa il piano del significante e giunge a quello del significato. Laccumulo delle "o», ben cinque, di cui quattro sotto accento, sembra evocare nel verso il concetto della meraviglia, sperimentata dal cuore che, circondato dall'infinito, dallo stupore trapassa allo stordimento. Siamo al centro esatto del testo, costituito di 15 endecasillabi; la pausa metrico-sintattica del punto fermo cade a metà del verso 8 , rendendo possibile la divisione del componimento secondo lo schema 7 endecasillabi + emistichio (un settenario: «il cor non si spaura.») + emistichio (un quinario: «E come il vento») + 7 endecasillabi. Si può osservare come l'accento forte, nel primo emistichio, cada sulla sesta sillaba, ossia proprio sulla vocale chiusa e scura «u»: quasi a imitare il colpo sordo del cuore che palpita chiuso e turbato nel proprio timore.

Dinanzi all' «apertura» degli spazi sconfinati fluttuanti come un'onda perpetua all'interno della mente che non può tutto quanto «abbracciare», l'io ha sperimentato la sensazione dello spaurimento. L'erranza oltre ogni confine metafisico, verso un punto di fuga di cui non si vede la fine, ha comportato l'impossibilità di trovare un appiglio, all'interno di sé, atto ad arginare le proiezioni potenzialmente illimitate dell'attività fantasmatica. Sul piano formale il punto fermo che divide in due emistichi il verso 8 riproduce quanto, sul piano contenutistico, è avvenuto nel corso dell'esperienza immaginifica: il cuore, percepita la paura di perdersi nell'infinito spaziale, ha avvertito la necessità di pausare il proprio tumulto. ${ }^{18}$

17. La nudità predicata del silenzio può significare metaforicamente la sua assolutezza e straordinarietà. Un "silenzio assoluto e straordinario» può, dunque, essere anche un «silenzio sovrumano".

18. Non sono d'accordo con chi equipara l'infinità dello spazio nella quale l'io si è trovato, "fingendo", con il nulla che spaventerebbe con il suo vuoto il soggetto poetante. Lo spazio che l'io si è trovato di fronte lo ha spaventato per la sua realtà: immateriale quanto si voglia ma "consistente», in quanto rappresentante una proiezione di sé. E il sé è l'antitesi del nulla. Il sé è il «pieno» dell'anima che si stupisce della propria «chiusura» rispetto all'infinita «apertura» degli spazi cosmici da essa immaginati. Cfr. Enrico FENZI, «Note di lettura all'"Infi- 
Dopo il punto fermo a metà del verso 8 il nuovo enunciato inizia con una congiunzione: «E come il vento / odo stormir tra queste piante [...]» (vv. 8-9). Come il «Ma» ha contrassegnato il punto di partenza dell'esperienza contemplativa dell'io, anche l' «E» sigla un nuovo inizio. L'io ha riconosciuto un suono familiare e tranquillizzante che proviene dal mondo esterno e intuisce in modo fulmineo che il proprio timore si inserisce nella vita che scorre perennemente e lo richiama ora dai silenzi infiniti al presente. Inizia con «E come» il nuovo presente e l'io, riattivata la pulsione vitale, può continuare la propria avventura nell'infinito. Non appena il soggetto ha udito «stormire» il vento fra «queste piante» —e il deittico si satura di significato, di pregnante vicinanzascatta il paragone fra "quello / infinito silenzio» (vv. 9-10), ancora impresso nella memoria, e "questa voce». Ora, tuttavia, il silenzio infinito è "quello", "quello», cioè, che il soggetto percepisce nello spazio circoscritto e familiare compreso fra "queste piante» e "questa voce». Il verso 10 accoglie fronte a fronte «infinito silenzio» e «questa voce»: l'antitesi, il «contrasto», fra «apertura» e «chiusura», «indefinito» e «finito» si ripropone, mentre l'io si avventura nella vastità dell'infinito procedendo in senso inverso rispetto a quanto ha fatto all' $i$ nizio. Il suono del vento fra le foglie è l'indicatore preciso del tempo presente da cui l'io non si distacca più nel suo percorso di immersione nel tempo passato. Dal paragone fra il silenzio assoluto ed il fruscio dell'aria tra le piante l'io si sovviene, ossia si rappresenta nella mente l'infinità temporale: «[...] l'eterno, / e le morte stagioni, e la presente / e viva, e il suon di lei» (vv. 11-13). In questo caso la presenza della consonante liquida «r», in combinazione con la sibilante «s», asseconda l'andamento del pensiero che si rappresenta lo scorrere del tempo attraverso i secoli, mentre la catena del polisindeto sembra moltiplicare sia la durata temporale, sia l'attività di un'immaginazione che nel passato retrocede e che dall'eterno riemerge:

Dolor mio nel sentire a tarda notte seguente al giorno di qualche festa il canto notturno de' villani passeggeri. Infinità del passato che mi veniva in mente, ripensando [...] ai tanti avvenimenti ora passati ch'io paragonava dolorosamente con quella profonda quiete e silenzio della notte, a farmi avvedere del quale giovava il risalto di quella voce o canto villanesco. (Zibaldone, Dic. 1818...8 Gen. $1820,50)$.

nito"», in $\Lambda A \Theta E B I \Sigma A \Sigma$, Ricordando Ennio S. Burioni, art. cit.: "Il moto di paura, dunque, sottolinea l'incombente presenza dei vertiginosi e indicibili orizzonti del nulla, oltre $\mathrm{i}$ modi con i quali il pensiero se li finge. Nello stesso momento, mentre fa questo, restituisce all'io, che in quel vuoto, per un attimo breve ma decisivo, ascolta il battito del suo cuore, l'intera verità del suo proprio tempo e quella del nulla che lo circonda», p. 107. Si tenga presente questo brano dello Zibaldone: «Perché questa (scil. la materia) ancorché ridotta a menomissime parti, una di queste minime particelle, è si può dir tanto lontana dal nulla, quanto tutta la materia o qualunque altra cosa esistente, cioè fra essa e il nulla, ci corre un divario e uno spazio infinito" (9 Feb. 1821, 631). Solo quando si sarà incupito il suo pessimismo filosofico, Leopardi scriverà: «Dove è nulla quivi è spazio, e il nulla senza spazio non si può dare» (Zibaldone, 14 Dic. 1826, 4233). 
Anche in questo brano dello Zibaldone è una "voce», un «canto» a innescare il processo di immersione nel più lontano passato che il giovane Leopardi paragonava con la quiete e il silenzio notturni, a loro volta percepiti con maggiore intensità perché il suono del presente funzionava come cassa di risonanza. Nell' Infinito, tuttavia, l'io, al termine del suo percorso non sperimenta una sensazione dolorosa, bensì una sensazione euforica e contesta di opposizioni: il piacere nasce dal contrasto fra l'entusiasmo del ritrovarsi ed il pregresso timore dello smarrimento, perché «l'anima riceve vita (se non altro passeggiera) dalla stessa forza con cui sente la morte perpetua delle cose, e sua propria» (Zibaldone, 3...4 Ott. 1820, 261).

La pausa forte a metà del verso 13 ha una valenza diversa rispetto a quella del verso 8 , ma ne trattiene la valenza, ne itera lo spessore significante e ne incorpora il significato: adesso ciò che risalta non è la paura del cuore, ma la vitalità di un suono che coincide con lo stormire del vento fra le foglie e il palpito rinnovato di un io che assapora la preziosità di uno spazio conosciuto e di un tempo «in atto» momentaneamente riconquistato.

Esiste un divario incolmabile fra passato e presente; un "contrasto" poetico e sublime fra la morte («le morte stagioni») e la vita (la stagione "presente») che risuona sfiorando le piante, nel momento in cui la propria immaginazione abbraccia l'infinito silenzio e insieme la parola del vento. Mentre il tutto si muove, nello spazio e nel tempo, l'io non prova più timore, tanto è intensa la percezione del piacere legato al «suon di lei»: della vita che "c'è», interiorizzata in uno spazio che è «silenzio», in un tempo che è «suono».

Lultimo fortissimo enjambement che separa aggettivo e sostantivo dilata la parola ossitona, chiara nel suo timbro vocalico aperto, «immensità»: e l'«inventore» è sul punto di ricomporre, con l'ultimo endecasillabo di senso compiuto, il "contrasto" fra finito $v s$ infinito, chiusura $v s$ apertura e terminare anche quel viaggio testuale affidato al suo idillio più intensamente poetico, nel quale il piacere che nasce appunto da un "contrasto" ha toccato uno dei suoi punti più alti. «Così, per ciò, «tra questa / immensità» (vv. 13-14) spaziotemporale -oltre la siepe, protetto dalla voce del vento- il pensiero del poeta può lasciarsi «annegare» e sprofondare in una dimensione senza confini: «e il naufragar mè dolce in questo mare». L'ultimo verso chiude circolarmente il componimento collegandosi al primo nel contenuto affettivo ('mi fu caro'; 'm'è dolce') e nel suono: «Sempre caro mi fu quest' ermo colle». E, circolarmente, dall' «oggetto comune» del "colle» si passa all'oggetto altrettanto consueto e familiare rappresentato dal «mare». E stato scritto che questo mare sia solo in parte metaforico e che mantenga qualcosa del suo significato letterale: prima di immergersi nelle sue fantasie Leopardi avrebbe trattenuto nella mente uno scorcio di quella lontana distesa che si estende a perdita d'occhio di fronte a Recanati. E vengono in mente i versi delle Ricordanze: 
di quel lontano mar, quei monti azzurri, che di qua scopro, e che varcare un giorno io mi pensava [...]. (Le Ricordanze, vv. 19-23)

Il mare è comunque accompagnato da un moto affettivo, da una sensazione dolce, in entrambi i contesti. Anche in quel passo, proprio all'inizio dello Zibaldone, in cui Leopardi - a proposito dei poeti antichi e al loro modo di comporre, così diverso rispetto a quello dei moderni, perché naturale e non artificiale, perché spontaneo, non affettato- scriveva: ${ }^{19}$ "ed ecco ottenuto dagli antichi il grand'effetto, che domandano i romantici, ed ottenuto in modo che ci rapiscono e ci sublimano e cimmergono in un mare di dolcezza [...]» (Ago. 1817 ...Dic. 1818, 16). Siamo molto vicini al verso di chiusura dell'Infinito: "e il naufragar mè dolce in questo mare». Figura dunque di un rapimento e di un inabissamento, di un elevarsi dell'animo sempre più in alto, poi giù, sino all'ossimoro sublime di sentirsi sprofondare in un dolce naufragio, in "quel» mare di dolcezza, in un mare-metafora equorea della poesia, antica e nuova: sembra essere anche questa la parabola disegnata da Leopardi nell'Infinito. Il viaggio conoscitivo, ondeggiante tra realtà e immensità, che si risolve nel finale abbandono, nella scomparsa dei limiti tra la realtà definita e l'infinito concepito dal pensiero, è insieme il viaggio della poesia, se, grazie alla sublime «immaginazione», il poeta oltrepassa la siepe, simbolo materiale e sensibile dell'ostacolo, dell'ombra che doveva essere attraversata per scoprire un'inesplorata regione dell'animo, per scorgere una bellezza nuova. E la dolcezza, contesta di infinite vibrazioni, è appunto l'effetto poetico che la parola del fanciullo cresciuto sui classici, eppure pervaso di straordinari spiriti romantici, presentifica e ripropone nella limpidezza di un itinerario della mente e del sentimento. Il portato finale di questo viaggio è il piacere infinito che si sprigiona da quella "commozione» che deriva dall'intreccio fra trasparenza della natura, vaghezza

19. Cfr. qui nota n. 8. Il brano dello Zibaldone così continua: «[...] quell'albero, quell'uccello, quel canto, quell'edifizio, quella selva, quel monte, tutto da per se, senz'artificio, e senza che questo monte sappia in nessunissimo modo di dover eccitare questi sentimenti, né ch'altri ci aggiunga, perché li possa eccitare, nessun'arte ec. ec. In somma questi oggetti, insomma la natura da per se e per propria forza insita in lei, e non tolta in prestito da nessuna cosa, sveglia questi sentimenti. Ora che facevano gli antichi? dipingevano così semplicissimamente la natura, e quegli oggetti e quelle circostanze che svegliano per propria forza questi sentimenti, e li sapevano dipingere e imitare in maniera che noi li vediamo questi stessi oggetti nei versi loro, cioè, ci pare di vederli, per quanto è possibile, quali sono in natura, e perché in natura ci destano quei sentimenti, anche dipinti e imitati con tanta perfezione ce li destano egualmente, tanto più che il poeta ha scelti gli oggetti, gli ha posti nel loro vero lume, e coll'arte sua ci ha preparati a riceverne quell'impressione, doveché in natura, e gli oggetti di qualunque specie sono confusi insieme, e in vederli spessissimo non ci si bada, [...] e bisogna poi perché producano quei tali sentimenti andarli a prendere pel loro verso: ed ecco ottenuto dagli antichi il grand'effetto che domandano i romantici, ed ottenuto in modo che ci rapiscono e ci sublimano e c'immergono in un mare di dolcezza, e tutte le età e tutti i secoli e tutti i grandi uomini e poeti che son venuti dopo di loro ne sono testimoni» (Zibaldone, 16). 
dell'immaginazione e prorompere del sentimento. Questo "commuovere», Leopardi, chiedeva alla vera poesia: ${ }^{20}$

e una poesia che lascia gli affetti de' lettori o uditori in pienissimo equilibrio, si chiama poesia? produce un effetto poetico? che altro vuol dire essere in pieno equilibrio se non esser quieti, e senza tempesta e commozione alcuna? E qual altro è il proprio uffizio e scopo della poesia se non il commuovere, così o così, ma sempre commuover gli affetti? (Zibaldone,16-18 Set. 1823, 3456).

20. Credo valga la pena di riportare questo brano dello Zibaldone, nonostante la lunghezza. Prendendo avvio da un'affermazione di Madame de Staël ne l'Histoire de Corinne, Leopardi scrive: «Bellissima condanna del sistema romantico che per conservare la semplicità e la naturalezza e fuggire l'affettazione che dai moderni è stata pur troppo sostituita alla dignità, (facile agli antichi ad unire colla semplicità che ad essi era sì presente e nota e propria e viva) rinunzia ad ogni nobiltà, così che le loro opere di genio non hanno punto questa gran nota della loro origine, ed essendo una pura imitazione del vero, come una statua di cenci con parrucca e viso di cera ec. colpisce molto meno di quella che insieme colla simplicità e naturalezza conserva l'ideale del bello, e rende straordinario quello ch'è comune, cioè mostra ne' suoi eroi un'anima grande e un'attitudine dignitosa, il che muove la maraviglia e il sentimento del contrasto, mentre nel romantico non potete esser commosso se non come dagli avvenimenti ordinari della vita, che i romantici esprimono fedelmente, ma senza dargli nulla di quello straordinario e sublime, che innalza l'immaginazione e ispira la meditazione profonda e la intimità e durevolezza del sentimento» (Zibaldone, Dic. 1818... 8 Gen. 1820, 86-87). 